\title{
Investigation of the siliceous hydrogel phase formation in glass-ionomer cement paste
}

\begin{abstract}
ABSTRCT
The microstructure evolution of a complex glass-ionomer cement (GIC) paste over the first $72 \mathrm{~h}$ of the cement setting reaction was investigated by small-angle neutron scattering (SANS) and infrared spectroscopy. GIC is a biocompatible material which is clinically used for dental fillings. In this study, GIC pastes were prepared, following the ISO9917-1:2007 cement preparation method, from medical grade poly(acrylic acid), $\mathrm{SiO}_{2}-\mathrm{Al}_{2} \mathrm{O}_{3}-\mathrm{P}_{2} \mathrm{O}_{5}-\mathrm{CaO}-$ $\mathrm{CaF}_{2}$-based fluoroaluminosilicate glass and $\mathrm{H}_{2} \mathrm{O} / \mathrm{D}_{2} \mathrm{O}$ solvent. During the setting reaction, polyacrylic acid attacks the fluoroaluminosilicate glass particles to form a siliceous hydrogel phase, glass core and hydrated polyacrylate matrix. The formation of the siliceous hydrogel structure and cross-linking of polyacrylate chains play important roles to harden the GIC. Infrared spectroscopy was used to identify the formation of the hydrogel phase and crosslinkage in GIC paste. In addition this paper reports SANS measurements for GIC pastes at different contrast conditions $\left(\mathrm{H}_{2} \mathrm{O}: \mathrm{D}_{2} \mathrm{O}\right.$ ratio) from the Bilby instrument at the Australian Centre for Neutron Scattering, ANSTO, Australia. The SANS data provide microstructure information for the hydrogel phase in GIC paste over the length scale of 10-5000 $\AA$.
\end{abstract}

Keyword: Small angle neutron scattering; Glass-ionomer cement; Fluoroaluminosilicate glass; Infrared spectroscopy; Setting reaction. 